\title{
Pharmaceutical compounding: Recent advances, lessons learned and future perspectives
}

\author{
Angeliki Siamidi, Natassa Pippa and Costas Demetzos* \\ Department of Pharmaceutical Technology, Faculty of Pharmacy, Panepistimioupolis Zografou, National and Kapodistrian University of Athens, Athens, Greece
}

\begin{abstract}
Pharmacists are considered as scientists that efficiently contribute in the health care system and their role is related to the protection of the community health. They are involved into the manufacturing process, by formulating a drug into a medicine based on the good compounding practice guidelines. This scientific direction and activity are considered as added values of their contribution into the efforts for improving the social profile of health systems. The Compliance Policy Guide (CPG) provides guidance to the pharmacists to bypass the unlicensed manufacturing process and to produce safe and effective medicines. The last is well adapted to the patients' medication needs based on personalized demands under a particular prescription by a physician. It is obvious that the pharmaceutical compounding is considered as the leading area of transforming high quality raw biomaterials into a medicine, for personalized health care. The pharmacists as compounders should follow all the guidelines that are required for producing safe and effective personal medications.
\end{abstract}

\section{Introduction}

\section{Pharmaceutical compounding}

According to the Food and Drug Administration (FDA), Pharmaceutical Compounding is defined as "a practice in which a licensed pharmacist, a licensed physician, or, in the case of an outsourcing facility, a person under the supervision of a licensed pharmacist, combines, mixes, or alters ingredients of a drug to create a medication tailored to the needs of an individual patient". According to Professional Compounding Centre of America (PCCA): "Pharmacy compounding is the art and science of preparing personalized medications for patients. Compounded medications are made based on a practitioner's prescription in which individual ingredients are mixed together in the exact strength and dosage form required by the patient. This method allows the compounding pharmacist to work with the patient and the prescriber to customize a medication to meet the patient's specific needs". In the United States Pharmacopeia Convention 795 [Pharmacy Compounding. (The United States Pharmacopeia 33 and National Formulary 28. Rockville, MD: United States Pharmacopeia Convention, Inc; 2010) the pharmaceutical compounding is defined as "the preparation, mixing, assembling, packaging, and labeling of a drug or device in accordance with a licensed practitioner's prescription, medication order, or initiative based on the practitioner/patient/ pharmacist/ compounder relationship in the course of professional practice".

Frequently, a community or a hospital pharmacist welcomes patients that cannot be able to accept an approved medication, prescribed by the physician. For example, patients suffering from allergic symptoms due to a certain excipient, elderly patients or child that can't swallow pills and need medicines in a liquid form that are not available in market, classified as patients that should be treated by personalized medicines. A compounding pharmacist with a physician's consent can prepare a unique drug dosage form, or a pharmaceutical vehicle, for a particular patient in order to minimize the undesirable effects of the medication, to adjust the therapeutic strength and or to alter the route of administration etc.

\section{The legislation of compounded medications}

As mentioned above, compounded dosage forms of drugs are not approved by regulatory agencies, which can not verify the efficacy, the effectiveness and safety of compounded medicines. There are several publications in the literature regarding the potential risks of Pharmacy Compounding and compounded medicines are characterized as highrisk level products for patient safety, especially for compounded sterile preparations [1-3]. Mullarkey (2009) described in her publication several issues surrounding pharmacy compounding, such as activities of compounding pharmacists, quality of compounded sterile preparations especially compounding errors (including purposeful potency alterations, unreported contamination etc.) and therapy issues [1]. There are several risks and liabilities of prescribing compounded medications (i.e. physician liability risk from prescribing compounding facility products, therapy management issues etc.) [3]. The FDA has begun to exert its considerable regulatory power by developing new guidelines and laws in the area of pharmaceutical compounding. Williams (2010) reported that pharmacists and compounders have objected to a variety of the agency's policies, resulting in acrimony, disputes, litigation, and a good deal of uncertainty regarding the rules of participation and tried to explain the nature of the controversy from the perspective of the litigation and discusses possible solutions to a confusing and an uncertain present [4]. Compliance Policy Guide (CPG), the stated intention was to provide guidance to pharmacists

Correspondence to: Costas Demetzos, Department of Pharmaceutical Technology, Faculty of Pharmacy, Panepistimioupolis Zografou, National and Kapodistrian University of Athens, Athens, Greece, E-mail: demetzos@pharm.uoa.gr

Key words: pharmaceutical compounding, pharmacists, compounded medications, legislation

Received: January 02, 2017; Accepted: January 27, 2017; Published: January 30, 2017 
as to how it intended to distinguish unlicensed manufacturing from compounding. The Compliance Policy Guide Pharmacy Compounding is obsolete and withdrawn in the $4^{\text {th }}$ of December 2013. For the above reasons, Drug Quality and Security Act (DQSA) was created and is a legislation (new law created in 2013) that contains important provisions relating to the oversight of compounding of human drugs.

\section{Pharmaceutical compounding and patient-centered healthcare}

Pharmaceutical compounding is synonymous to patient centered services with an added value for the consumers. According to McPherson et al. (2013) compounded medicines enhance pharmacist contribution to developing and implementing patient therapeutic plans and provide customized medications of high pharmaceutical quality [5]. For the regulatory point of view there is a debate in the literature concerning the pharmaceutical compounding and manufacturing $[6,7]$. The application and implementation with Quality by Design concepts to the preparation/ manufacturing process for a compounded medication can help in understanding the potential pitfalls; ameliorate the quality of the final compounded products and the means to mitigate their impact [8].

\section{The leader role of pharmacist}

Additionally, the role of pharmacist is central in the Health Systems. Drug-related problems and adverse drug reactions are usually identified and reported by community pharmacies [9]. For example, the identification, resolution, and documentation of drug related problems are central to community pharmacy practice in Sweden and a national drug related database, patient medication profiles, and a new national register of medicines dispensed to patients provide opportunities for growth in pharmaceutical care practice and research in this country [10]. This task is also included to the pharmaceutical care that is provided from community pharmacies in many countries i.e. UK, Canada and USA [11-13]. On the other hand, according to Christensen and Farris; 2006 in the UK, "Dispensing of prescriptions is the primary duty of community pharmacies and remains their core activity. Pharmacists dispense medicines at the order of a prescriber, typically a physician but possibly a physician assistant, nurse practitioner, optometrist, or dentist, as well. While community pharmacies are the main source for filling prescriptions for most patients, other sources include hospitals, health maintenance organizations, community clinics, and Internet mailorder pharmacies." There is a strong interplay between pharmaceutical compounding and pharmaceutical care. Yancey et al., 2008 described a closer relationship of a pharmacist with patients receiving compounded preparations than with patients receiving only manufactured products because higher compounded prescription volume was associated with a higher level of pharmaceutical care services for consumers receiving compounded medications [14].

Compounding pharmacists usually work as traditional pharmacists, with an emphasis on customizing medication according to patient's or physician's needs, therefore, they must hold a Pharmacy Degree and be licensed in their country of practice. Apart from their regular duties that include filling prescriptions, consulting with patients, overseeing pharmacy technicians, etc., compounding pharmacists have the added task of ensuring availability of the laboratory equipments, raw materials, medications, ingredients and supplies needed for compounding but also have the special skills needed to produce medications that are not commercially available. Pharmacists around the world have completed a compounding course as part of their core curriculum in their university program and have at least a basic knowledge of compounding pharmaceuticals upon graduation and therefore are not required to have any more licensing or certification than traditional pharmacists although, several organizations have proposed different level certification for compounders e.g., pharmacists would pass different theory and skills exams to compound topical versus oral versus sterile preparations [15]. However, at this moment, pharmacists that wish to work specifically in compounding are not obliged, but can choose, to receive more extensive training and accreditation. This additional and specialized training in compounding is usually offered by several professional organizations and provide training on how to compound tablets, capsules, oral suspensions, solutions, topical creams, gels, suppositories, sterile preparations, etc. following the appropriate guidelines.

\section{Specialized compounding}

The role of traditional compounding has declined due to the availability of mass-manufactured medicines but over the past years specialized compounding is increasingly offered from pharmacies [16]. This "rebirth" of compounding to each specialized form (i.e., a medicine designed especially for an individual patient) added value to the pharmacist's scientific identification. The additional interaction with medical practitioners and patients compared with routine dispensing as well as the involvement in the production of customized medications transforms the pharmacist from a supplier of medicines to an appreciated researcher in the scientific community [17]. Pharmacists have the deep knowledge of medicinal compatibilities needed to combine one or more chemicals into a new preparation or process the existing dosage form into one that is better suited to the patient's needs. On the other hand, medical health care providers should be aware on the evolution in the prescriptions practice taking into account the revolution in material science. It is the responsibility of pharmacists to be updated in the technological advances and by working closely with prescribers, to inform them of the new technological aspects and to choose together the best therapy options for patients. This project can positively affect social health and consequently health systems will recognize the importance of compounding and provide for its continued practice.

The comparison of compounding and non-compounding pharmacists' perceptions of the economic value of compounded preparations relative to commercial products are described in the recent study of Lobb et his collogues (2015) [18]. This research aimed to understand in deep the perception of pharmacists of areas for potential direct and indirect economic cost savings as a result of pharmaceutical compounding. On the other hand, the compounding and non-compounding pharmacists' ratings were significantly different. Compounding pharmacists more strongly believed that compounded medicines offered benefit in the human's well-being and the patient centered healthcare system [18]. Moreover, the economic value and the added-value of compounding and commercial pharmaceuticals cost savings associated strongly with compounded medications [18]. Pharmaceutical compounding also plays a key role in pharmacistpatient communication and affecting their professional status $[16,19]$. The reprofessionalisation strategy of the pharmacists in the competitive area of our society could also be based on pharmaceutical compounding and on the development of strong relationships with patients [16]. Pharmacists belong to the core of the healthcare environment as they are the most easily accessible health professionals. The community pharmacy and the pharmaceutical compounding are the leading areas for the symbolic transformation of the drug into medicine, which is strongly related to the transformation of excipients to biofunctional 
biomaterials, to the development of personalized medicines and to patient -centered healthcare systems [16].

Giam et al. [17] in their investigations reported very serious findings that 1 . Pharmacists providing specialized compounding reported that compared with their prior routine practice, they used a greater variety of ingredients and dosage forms for more clinical conditions, used different equipment and processes, and had upgraded facilities for handling compounded products 2 . Consumers and medical doctor interactions were more involved and in the case of physicians, more collaborative compared with routine dispensing (in other words greater collaboration and interaction with prescribers and patients) and 3. The pharmacists feeling more empowered in their roles, with improved professional satisfaction. An evaluation of the integration of standards and guidelines in community pharmacy practices are also appeared in the literature [20]. A lack of integration of practice standards indicated a need to review the standards for relevance and the majority of pharmacists believe that there is a need to re-evaluate flowchart models (workflow redesign) and procedures based on new roles, guidelines and responsibilities [20,21].

\section{Compounding errors}

Reclaiming pharmaceutical compounding as a specialization is surely an attempt of pharmacists to regain control of this core function but there are calls for regulatory and practice standard changes. Recent concerns about appropriate and safe compounding practices, both in community pharmacies but in hospital pharmacies as well, have been addressed [22]. According to the research in [2] "while drugs manufactured and tested in accordance with GMP regulations cannot be guaranteed to always be free of quality problems, the probability that FDA-approved drugs will consistently meet required quality standards is higher than it is for compounded drugs". In the unfortunate event when a preparation error occurs in pharmacy compounding, only a limited number of patients will be affected. As mentioned previously [23], in most cases the threats to patient safety that compounded medications pose are mainly due to: 1 . quality (e.g., product identification, purity, stability, compatibility), 2. the environment (e.g., using a segregated compounding area with specialized airflow capabilities, reducing particulate matter, practicing proper hygiene, properly cleaning of equipment and work areas), 3. personnel activities (e.g., human error, familiarity with protocols, following expiration dates), and 4 . the control process (e.g., process monitoring, control test post preparation). As pharmaceutical companies comply with GMP regulations to reduce the level of risk inherent in the large-scale production of drugs, so pharmacies that perform compounding in the small-scale preparation of medicinal products should comply with GLP regulations governing every aspect of drug preparation and testing. Compounding pharmacies should implement and maintain the protocols stated in the United States Pharmacopoeia under the chapters <795> Pharmaceutical compounding, for non-sterile preparations and $<797>$ Pharmaceutical compounding, for sterile preparations, to achieve consistent high quality and minimize errors. It is the compounding pharmacists' obligation to ensure adherence to these standards for compounding that will finally lead to the desirable medical care.

\section{Conclusion}

Pharmaceutical Compounding is the practice and the science of preparing personalized medications for patients. Compounded medicines enhance pharmacist contribution to developing and implementing patient therapeutic plans and provide customized medications of high pharmaceutical quality. The aim of this article was to present the leader role of pharmacist for the patient-centered healthcare system based on compounded medicines. Pharmacists should have a deep knowledge of specialized compounding, new protocols and regulatory updates in order to bridge the gap between the needs of the patients and the legislation of compounded medications.

\section{References}

1. Mullarkey T (2009) Pharmacy compounding of high-risk level products and patient safety. Am J Health Syst Pharm 66: S4-13.

2. Gudeman J, Jozwiakowski M, Chollet J, Randell M (2013) Potential risks of pharmacy compounding. Drugs R D 13: 1-8.

3. Randell MD, Duffy PJ (2014) Risk and liabilities of prescribing compounded medications. Postgrad Med 126: 178-180.

4. Williams KG (2010) Regulation of compounding by the food and drug administration: a tale of 2 circuits. J Pharm Pract 23: 502-506.

5. McPherson TB, Fontane PE, Day JR (2013) Patient-centered care as value-added service by compounding pharmacists. Int J Pharm Compd 17: 339-343.

6. Guharoy R, Noviasky J, Haydar Z, Fakij MG, Hartman C (2013) Compounding pharmacy conundrum: "we cannot live without them but we cannot live with them" according to the present paradigm. Chest 143: 896-900.

7. Timko RJ, Crooker PE (2014) Pharmaceutical compounding or pharmaceutical manufacturing? A regulatory perspective. Int J Pharm Comp 18: 101-111.

8. Timko RJ (2015) Applying Quality by Design Concepts to Pharmacy Compounding Int J Pharm Compd 19: 453-463.

9. Hämmerlein A, Griese N, Schulz M (2007) Survey of drug related problems identified by community pharmacies. Ann Pharmacother 41: 1825-1832.

10. Westerlund LT, Bjork HT (2006) Pharmaceutical care in community pharmacies: practice and research in Sweden. Ann Pharmacother 40: 1162-1169.

11. Jones EJ, Mackinnon NJ, Tsuyuki RT (2005) Pharmaceutical care in community pharmacies: practice and research in Canada. Ann Pharmcother 39: 1527-1533.

12. Noyce PR (2007) Providing patient care through community pharmacies in the UK policy, practice and research. Ann Pharmcother 41: 861-868.

13. Christensen DB, Farris KB (2006) Pharmaceutical care in community pharmacies: practice and research in the US. Ann Pharmacother 40: 1400-1406.

14. Yancey V, Yakimo R, Perry A, McPherson TB (2013) Perceptions of pharmaceutical care among pharmacists offering compounding services. J Am Pharm Assoc 48: 508-514.

15. Newton WD (2003) Compounding Paradox: Taught Less and Practiced More. Am J Pharm Educ 67: 12-14

16. Giam JA, McLachlan AJ, Krass I (2011) Community pharmacy compounding-impact on professional status. Int J Clin Pharm 33: 177-182.

17. Giam JA, McLachlan AJ, Krass I (2012) Characterizing specialized compounding in community pharmacies. Res Social Adm Pharm 8: 240-252.

18. Lobb WB, Wilkin NE, Holmes ER (2015) Pharmacists' perceptions of the economic value of compounded pharmaceuticals: A comparison of compounded and commercial pharmaceuticals in select disease states. Int J Pharm Compd 19: 514-520.

19. Shah B, Chewning B (2006) Conceptualizing and measuring pharmacist-patient communication: a review of published studies. Res Social Adm Pharm 2: 153-185.

20. Hattingh HL, King MA, Smith NA (2009) An evaluation of the integration of standards and guidelines in community pharmacy practices. Pharm World Sci 31: 524-549.

21. Angelo LB, Ferreri SP (2005) Assessment of workflow redesign in community pharmacy. J Am Pharm 45: 145-150.

22. Drazen JM, Curfman GD, Baden LR, Morrissey S (2012) Compounding Errors. N Engl J Med 367: 2436-2437.

23. Hicks RW (2014) Understanding medication compounding issues. AORN Journal 99 466-479.

Copyright: (C2017 Siamidi A. This is an open-access article distributed under the terms of the Creative Commons Attribution License, which permits unrestricted use, distribution, and reproduction in any medium, provided the original author and source are credited. 